OPEN ACCESS

Edited by:

Lucia Beccai,

Fondazione Istituto Italiano di

Technologia, Italy

Reviewed by:

Stefano Palagi,

Max Planck Institute for Intelligent

Systems, Germany

Federico Carpi,

Queen Mary University of London,

UK

*Correspondence: Marcello Calist

m.calisti@sssup.it

Specialty section:

This article was submitted to Soft Robotics,

a section of the journal

Frontiers in Robotics and Al

Received: 28 June 2016 Accepted: 07 September 2016 Published: 26 September 2016

Citation:

Calisti M, Cianchetti M, Manti M, Corucci F and Laschi C (2016) Contest-Driven Soft-Robotics Boost: The RoboSoft Grand Challenge. Front. Robot. Al 3:55. doi: 10.3389/frobt.2016.00055

\section{Contest-Driven Soft-Robotics Boost: The RoboSoft Grand Challenge}

\author{
Marcello Calisti ${ }^{1 *}$, Matteo Cianchetti ${ }^{1}$, Mariangela Manti ${ }^{1}$, Francesco Corucci ${ }^{1,2}$ and \\ Cecilia Laschi ${ }^{1}$ \\ ${ }^{1}$ The BioRobotics Institute, Scuola Superiore Sant'Anna, Pontedera, Italy, ${ }^{2}$ Morphology, Evolution and Cognition Lab, \\ University of Vermont, Burlington, VT, USA
}

This paper reports the design process, the implementation, and the results of a novel robotic contest addressing soft robots, named RoboSoft Grand Challenge. Applicationoriented tasks were proposed in three different scenarios where soft robotics is particularly lively: manipulation, terrestrial, and underwater locomotion. Starting from about 60 expressions of interest submitted by international teams distributed across the world, 19 robots were eventually selected to participate in the challenge in two of the initially proposed scenarios, i.e., manipulation and terrestrial locomotion. Results highlight both the effectiveness and limitations of state of the art soft robots with respect to the selected tasks. The paper will also focus on some of the advantages and disadvantages of contests as technology-steering mechanisms, including what we called "reductionist design," a phenomenon in which simplistic solutions are devised to purposely tackle the proposed tasks, possibly hindering more general and desired technological advancements.

Keywords: soft robotics, robot manipulation, robot locomotion, robotic challenge, robot competition

\section{INTRODUCTION}

The Olympic creed: "The most important thing in life is not the triumph, but the fight; the essential thing is not to have won, but to have fought well." has ubiquitous consensus in sport as well as in robotic contests. Challenging long-term goals foster innovation, from single components to system integration procedures (Masters and Delbecq, 2008), regardless of the success of each specific robot during the competition. Moreover, hands-on competitions have significant educational potential at all levels of academic training, from undergraduate to graduate courses (Murphy, 2000; Wyffels et al., 2011). In the past decades competitions and challenges spread over several domains of robotics (Dias et al., 2016); an heterogeneous (yet not exhaustive) list is presented in Table 1 and includes the renown Darpa Robotic Challenge (DRC) (Guizzo and Ackerman, 2015), euRathlon (Schneider et al., 2015), RoCKIn (Lima et al., 2014), European Robotic Challenges (EuRoCs), Mohamed Bin Zayed International Robotic Challenge (MBZIRC), the UAE drones for good award, RoboCup (Kitano et al., 1998), Cybathlon (Riener and Seward, 2014), to mention a few.

Often based on multi-disciplinary domains, robotic contests stimulate advancements in different areas, from hardware to algorithmic development. It is, however, possible to classify these events in two broad categories: competitions and challenges. Competitions are more similar to sport events, where well-defined rules allow to compare performance of teams (possibly encompassing both robots and humans) in tasks that are generally already solved by the state of the art technology. An example of competition is the RoboCup Soccer: Small Size category, where well-defined wheeled vehicles move in a soccer-like playground with the purpose of scoring more goals than the opponent team. On the 
TABLE 1 | A list of events related to robotics, with the aims declared by organizers, labeled as challenges and competitions.

\begin{tabular}{|c|c|c|c|}
\hline Event name & Aim & Address to & Type \\
\hline DRC (Darpa Robotic Challenge) & To catalyze focused efforts to spur innovation in robotics & Companies and academia & Challenge \\
\hline euRathlon & To challenge intelligence and autonomy of robots in realistic mock scenarios & $\begin{array}{l}\text { Universities, research labs, } \\
\text { or industry }\end{array}$ & Competition \\
\hline $\begin{array}{l}\text { Mohamed Bin Zayed International } \\
\text { Robotic Challenge (MBZIRC) }\end{array}$ & To demonstrate the current state of the art and to inspire the future of robotics & $\begin{array}{l}\text { Universities, research labs, } \\
\text { or industry }\end{array}$ & Challenge \\
\hline RoboCup & $\begin{array}{l}\text { To promote robotics and Al research, by offering a publicly appealing, but } \\
\text { formidable challenge }\end{array}$ & Companies and academia & Competition \\
\hline RoCKIn & $\begin{array}{l}\text { To foster scientific progress and innovation in cognitive systems and robotics } \\
\text { through the design and implementation of competitions }\end{array}$ & Mainly universities & Competition \\
\hline EuRoCs & $\begin{array}{l}\text { To strengthen collaboration and cross-fertilization between the industrial and the } \\
\text { research community via three industrially relevant challenges }\end{array}$ & Companies and academia & Challenge \\
\hline Cybathlon & $\begin{array}{l}\text { To facilitate conversation between academia and industry, to facilitate discussion } \\
\text { between technology developers and people with disabilities, and to promote the } \\
\text { use of robotic assistive aids to the general public }\end{array}$ & Companies and academia & Competition \\
\hline UAE drones for good award & $\begin{array}{l}\text { To find solutions that will improve peoples lives and provide positive technological } \\
\text { solutions to modern day issues }\end{array}$ & Companies and academia & Competition \\
\hline ELROB & $\begin{array}{l}\text { To demonstrate and compare the capabilities of unmanned systems in realistic } \\
\text { scenarios and terrains }\end{array}$ & Companies and academia & Competition \\
\hline ESA Lunar Robotic Challenge & $\begin{array}{l}\text { To gain a valuable insight into the challenges astronauts will face when operating } \\
\text { robots on the lunar surface }\end{array}$ & Academia (students) & Challenge \\
\hline HuroCup & $\begin{array}{l}\text { To emphasizes the development of flexible, robust, and versatile human-like robots } \\
\text { that can perform many different tasks in different domains }\end{array}$ & Academia (students) & Competition \\
\hline SAUC-E & $\begin{array}{l}\text { To design and build an autonomous underwater vehicle (AUV) capable of } \\
\text { performing realistic missions }\end{array}$ & Academia (students) & Competition \\
\hline SAUVC & $\begin{array}{l}\text { To provide students an opportunity to experience the challenges of AUV system } \\
\text { engineering and develop skills in the associated technologies }\end{array}$ & Academia (students) & Competition \\
\hline First Lego League & To involve kids in engineering and scientific work & Academia (students) & Competition \\
\hline
\end{tabular}

This list is not complete, and it is just intended to offer an overview of the different challenges/competition in several diverse domains.

other hand, robotic challenges are usually task-oriented events that propose activities at the very edge of the state of the art: an example is the DRC, where robots are demanded to move and operate in unstructured scenarios. It is not uncommon, in challenges, to observe robots obtaining a null score in one or more tasks, despite the quality of the implemented technical solution.

Despite the specificities of each robotic challenge, we can identify three general common objectives. The first one is to push forward the state of the art, promoting new ways to solving the proposed tasks, or to approach the development of the required technical solutions; the second one is to steer and canalize technological development toward the solution of significant societal and industrial challenges, with the aim of increasing the impact of robotic research (Masters and Delbecq, 2008); the third one is to enable the benchmarking of different robotic solutions through task-based scoring systems, which allow to assess and compare extremely heterogeneous and complex systems, such as robots (Ferri et al., 2015). These wide-range goals are particularly important for emerging fields, which often experience rapid development in conceptually different directions: this is the case for Soft Robotics.

Soft robotics is a growing and highly multi-disciplinary field, which proposes to endow robots with unprecedented capabilities not only through sophisticated control systems but also by exploiting material and morphological properties, incorporating the use of soft materials into robotics control. This allows to alleviate the computational burden of the controller and to better cope with unpredictable unstructured environment, like in search and rescue and disaster response operations, as well as in medical and human assistance tasks (Kim et al., 2013; Laschi and Cianchetti, 2014). This ground-breaking approach requires to rethink all the conventional stages of robot development, from design to fabrication and, finally, control (Rus and Tolley, 2015): in order to better cope with these dares, coordinated efforts are desirable.

Under the Future and Emerging Technologies scheme, the European Commission funded a Coordination Action (CA) called RoboSoft, with the aim of assisting soft robotics researchers to combine their endeavors to maximize the opportunities and to materialize the huge potential impact of this field. Within the framework of the RoboSoft CA, a competitive event regarding soft robots appeared timely to pursue the above-mentioned general objectives of robotic challenges in the specific context of soft robotics, as well as to demonstrate the capabilities of state of the art soft machines in realistic mock environments. Therefore, the RoboSoft Grand Challenge was developed as an instrument to show the potential of soft robotics to push forward the state of the art of this discipline, and to foster collaborations among different research groups worldwide. To the best of authors' knowledge, no challenge existed before that was specifically designed for soft robots. The only competition devoted to soft robotics is the initiative of the Soft Robotics Toolkit (SRT) 
(SoftRoboticsToolkit, 2016), where open-ended designs can be submitted for evaluation by a panel of qualified judges. In the 2015-2016 editions of the competition, entries had to use at least one technological element of the SRT and 2/5 - 2/5 of the score was assigned to the design and documentation, while $1 / 5$ was assigned to the implementation and actual demonstration. Conversely to the SRT competition, the RoboSoft Grand Challenge (RSGC) aims to enhance and showcase the capabilities of soft robots in realistic mock scenario, with the ambitious aim of demonstrating the added value of soft robots in terms of interaction with the environment, adaptability, and resilience. On the one hand, RSGC was designed to demonstrate that soft robots are capable of performing traditional robotics duties (e.g., pick and place); on the other, a goal was to show how soft robots can exploit softness to accomplish tasks that are very difficult for their rigid counterpart (e.g., manipulation of fragile objects, adaptation to the environment).

\section{DESIGN OF THE RoboSoft GRAND CHALLENGE}

\subsection{Event Definition}

The first edition of the RSGC was a task-oriented challenge, where the robots were asked to solve not only tasks that traditionally appear in robotic competitions but also some that were purposefully designed. Indeed, being devoted to highlighting soft robots capabilities - which have not been challenged so far - scenarios and tasks could not be entirely drawn from other challenges, but had, in some cases, to be designed entirely from scratch. Another difficulty that had to be taken into account lies in the heterogeneity of the soft robotics field, which encompasses very different, and rapidly evolving, platforms and technologies for what concerns actuation, fabrication, sensing, and control. These aspects increased the difficulty of defining the challenge, which had to be a good trade off between being feasible (to showcase the state of the art and encourage a wide participation) and arduous (to stimulate the field). To solve this design conflict, an extensive overview of state of the art soft robots was performed, followed by an analysis of the most commonly required tasks in current robotics challenges/competitions. In some cases, these tasks were reinterpreted to adjust the difficulty level to the state of the art of soft robots. In others, new tasks were devised ad hoc in order to promote the adoption of soft technologies and challenge soft robots with tasks that they are expected to be able to solve, that are, in turn, extremely difficult - or even impossible - for completely rigid machines.

In order to respect the heterogeneity of the field, we did not define by regulation what kind of soft robots could compete in the challenge, which would have restricted the participation to those robots only that are compliant with our definition of a soft machine. Soft robotics includes very different technological solutions, exploiting e.g., shape memory alloys (Seok et al., 2013), silicone rubber and pneumatic actuation (Shepherd et al., 2011), tendon-driven actuation (Arienti et al., 2013), tensegrity mechanisms (Kim et al., 2014), vibration and deformation of alloys (Yu and Iida, 2014), granular jamming (Steltz et al., 2009), and many more, thus, that a focused definition would also be arduous. Instead, we pushed on the capabilities which we consider fundamental for the soft robots, namely resilience, mechanical compliance, and delicate interaction with the environment, together with strength and dexterity. Scenarios and tasks were, thus, designed to highlight soft robots features and skills, without imposing rules on the design and technologies adopted to realize the robots. The only constrains imposed to participants are related to logistics (i.e., maximum power supply available, security rules, etc.) and about the maximum size and weight of the robots. Dimensions influence the design of the whole competition field, so it had to be known a priori. Since robot miniaturization was not considered a crucial aspect in this moment, a comfortable bounding cube of side $0.6 \mathrm{~m}$ was selected, which already includes most of the existing soft robots.

Another general remark concerns autonomy. Several events stress or directly aim at increasing robots autonomy (both in the sense of embedding all subsystems into the robot - e.g., have robots carrying their own power supply and actuation systems - and to increase autonomous operation). As an example, RoboCup Soccer Leagues require each robot to be autonomous, and the team is required to act as a whole in order to realize an effective and coordinated behavior, which requires sophisticated control algorithms, while organizers of euRathlon report that autonomous operations are far from being perfect among the competing robots, and aim at increasing its importance in future editions. Autonomy is certainly a key issue to the success of robotics, and this kind of challenges/competitions - in which consolidated platforms and technologies are adopted to realize the competing robots - are better suited to stimulate development in this direction. Soft robotics, however, is a young niche of robotics, and many challenges are still to be solved for what concerns basic technologies for robot mechanical design, sensing, and actuation. Also, morphology and control are tightly coupled in these machines, which pose additional challenges. Finally, most soft robots require to date external equipment to operate (power supply, electronics, actuation systems, etc.). In some cases miniaturizing and embedding these equipments into the robots is not a technical issue, but only a monetary one, which we would not enforce to participants. Thus, the RSGC did not impose strong constraints or rewards related to the autonomy of the competing robots: nevertheless, we will see in the tasks implementation in Section 2.3 and in the scoring systems in Section 3 how tethered robots are, implicitly, slightly penalized. With this overall view of the event, we now describe the actual scenarios where the robots competed.

\subsection{Scenarios Definition}

To define suitable domains and scenarios for the RSGC, we performed a deep survey of the state of the art of soft robotics. This discipline is steeply growing, and from the seminal review paper of Trivedi et al. (2008) the field was subject to several evolutions; to date, the most recent review paper on soft robotics (Rus and Tolley, 2015) identifies four possible application domains for soft robots: locomotion, manipulation, wearable, and soft cyborgs. This review agrees with our survey on the most relevant domains influenced by soft robotic, which identified three niches: (1) the 
terrestrial locomotion, where a great number of bio-inspired (Belanger et al., 2000; Mezoff et al., 2004; Lin et al., 2013; Umedachi et al., 2016) (inspired by worms, caterpillars, and their gaits) (Jayaram and Full, 2016) (insects) (Chrispell et al., 2013; Cicconofri and DeSimone, 2015) (snakes) or build from scratch robots (Kim et al., 2014; Li et al., 2016) are under development; (2) the underwater locomotion (Fiazza et al., 2010), mainly inspired by fishes (Clark et al., 2015), turtles (Song et al., 2016), crabs (Calisti et al., 2016), chephalopods (Arienti et al., 2013; Cianchetti et al., 2015), rays (Urai et al., 2015), or other aquatic animals; and (3) manipulation, either at the level of grippers (Manti et al., 2015; Fakhari et al., 2016; Shintake et al., 2016), arms (Cianchetti et al., 2014; Elango and Faudzi, 2015; Katzschmann et al., 2015; Deashapriya et al., 2016; Sun et al., 2016), or other devices (Deng et al., 2016).

The selected domains were declined in three realistic mock scenarios: a terrestrial race, an underwater race, and a manipulation field. Inside each domain, there are several possible implementations, for example, the terrestrial race could be a sprint competition, the underwater race could be based on Simultaneous Localization and Mapping (SLAM), the manipulation could be related to industrial environment and so on. To cover as much as possible the potential applications of soft robotics, we decided to implement complex mock scenarios which allowed the robot to show several capabilities: the terrestrial race was based on a simulated emergency situation, where the robot was demanded to move over unstructured, fragile, and unstable environments; the underwater race was based on a navigation and interaction scenario, where the robot was demanded to precisely move through waypoints, then gently (but firmly) interact with the environment in order to remove some objects; finally, the manipulation scenario replicated structured environments, in which robots had to demonstrate both dexterity, gentle interaction, and strength. A resuming picture of the features that were expected in the various scenarios is presented in Figure 1. Each scenario was split-down into tasks, whose scoring and evaluation mechanisms are designed in order to capture the expected capabilities of the robots.

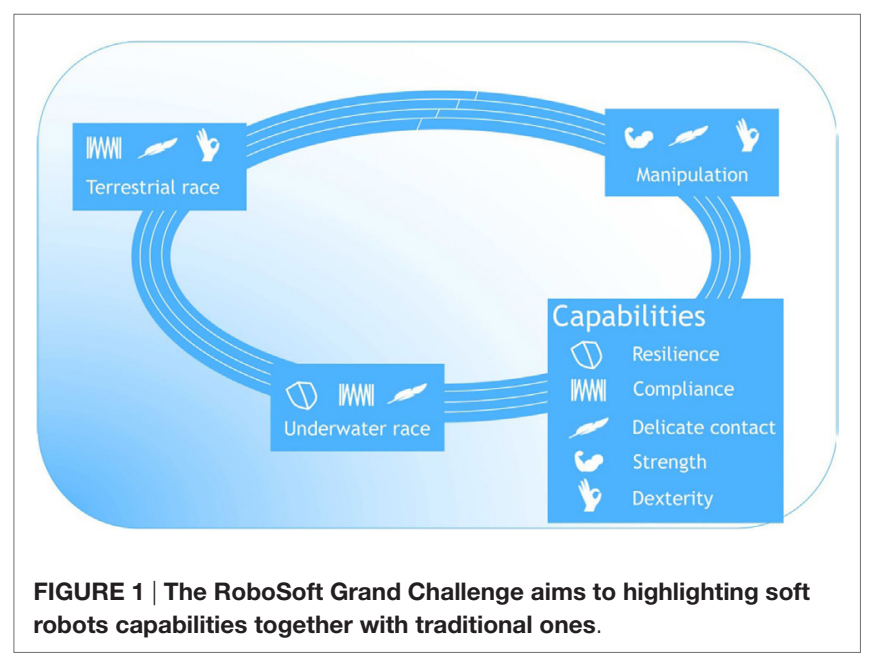

\subsection{Tasks Definition}

Task definition is the core phase of the challenge design. We considered four aspects/features during tasks selection:

1. soft-related capabilities to be shown

2. scenario-related traditional tasks

3. traditional/soft-related tasks relationship

4. feasibility/challenge trade-off

The selection process then took four consecutive steps, which would possibly iterate upon evaluation. To begin with, soft-related tasks were proposed with the aim of highlighting specific capabilities of soft robots. Envisaged tasks were (s1) jump from high positions; (s2) reduce body dimension; (s3) adapt to the environment; (s4) perform delicate contact; and (s5) gently manipulate (grasp/move/interact) objects. Subsequently, traditional scenario-related tasks were proposed to highlight the performance of soft robots against common robotic duties. In the scenarios we wanted to develop, traditional tasks were individuated as: ( $\mathrm{t} 1$ ) move over uneven terrains; ( $\mathrm{t} 2$ ) move over obstacles (stairs/debris); ( $\mathrm{t} 3$ ) exert forces to the environment; and (t4) manipulate complex (size/weight/shape) objects. Then the relationships among soft and traditional tasks were taken into account, with the goals of avoiding the redundant demonstration of the same capabilities, and increase the complexity of the challenge with conflictual tasks, i.e., soft task (s4) - delicate contact could be in contrast with the traditional ( $\mathrm{t} 3$ ) - exert forces to the environment, while (s2) - reduce body dimension could be in contrast with ( $\mathrm{t} 2)$ - move over obstacles. This process led to formalizing three fields for each scenario, which were then qualitatively evaluated by predicting the performance of state of the art robots in those tasks. Although this qualitative evaluation was mainly inductive, it guided us in tuning the complexity level of the tasks, so that desired advancements with respect to the state of the art could be within the reach of the teams.

After this process, the terrestrial locomotion scenario (locomotion race) was proposed with the following tasks:

1. A sand box representing the ground outside a collapsed building, a traditional locomotion task.

2. A sliding aperture representing a narrow aperture in a building that the robot should enter, demonstrating shrinking capabilities.

3. A stair that the robot should negotiate, moving both upward and downward.

4. A congested, unstable environment (common in search and rescue and disaster response scenarios) which could collapse if the robot exerted too much force onto the structural elements.

The three-dimensional model of the terrestrial race can be seen in Figure 2, together with the actual implementation: the overall dimensions of the field were about $9 \mathrm{~m}$ length and $2 \mathrm{~m}$ wide. Teams were allowed to skip some tasks and move directly to the next one, but were not allowed to go back. The manipulation scenario was structured in the following tasks: 


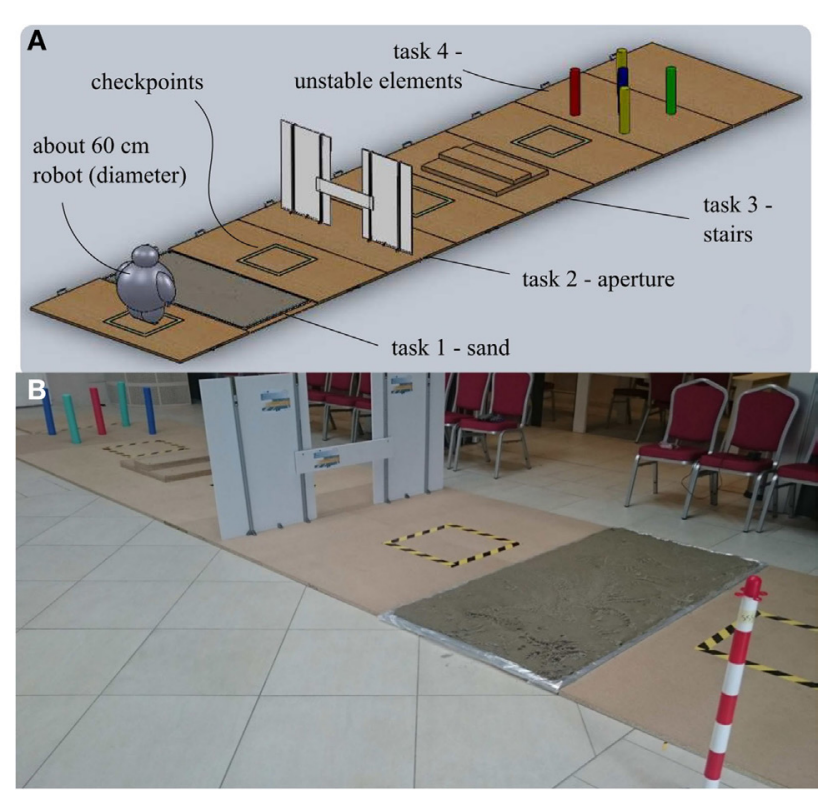

FIGURE 2 | The locomotion scenario was made of four tasks separated by checkpoint tiles. Picture (A) shows the CAD model, picture (B) shows the actual implementation.

1. Pick and place of different objects

2. Arm positioning

3. Door opening

The objects in the first task were selected to highlight gentle contact and adaptability, while the arm positioning task required also dexterity. The door opening is a classical manipulation task, which jointly demonstrates strength and dexterity. This scenario was implemented as a structured environment, shown in Figure 3. Teams had two options to cope with the tasks: in the first one, the (mobile) robot moved itself toward the manipulation area and performed the demanded tasks. In the second option, teams were allowed to attach their robots to a linear slider. In this scenario, teams were allowed to tackle tasks in the order they prefer. Finally, the underwater race scenario encompassed the following tasks:

\section{A navigation task}

2. A sliding aperture the robot should enter

3. A manipulation task, where some objects should be removed from the ground of the pool

The robot was deployed from a platform about $50 \mathrm{~cm}$ above the water. The robot should be resilient to this small "jump," thus demonstrating easy deployment. The first task of this scenario required to navigate toward two buoys placed at different heights from the bottom of the pool, which is a traditional navigation task. The second task was to move inside an aperture that can be reduced or increased in dimension, thus demonstrating squeezing capabilities, while the third task required to remove the infesting algae without dislodging fragile elements (such as amphorae and corals). A depiction of the field model is presented

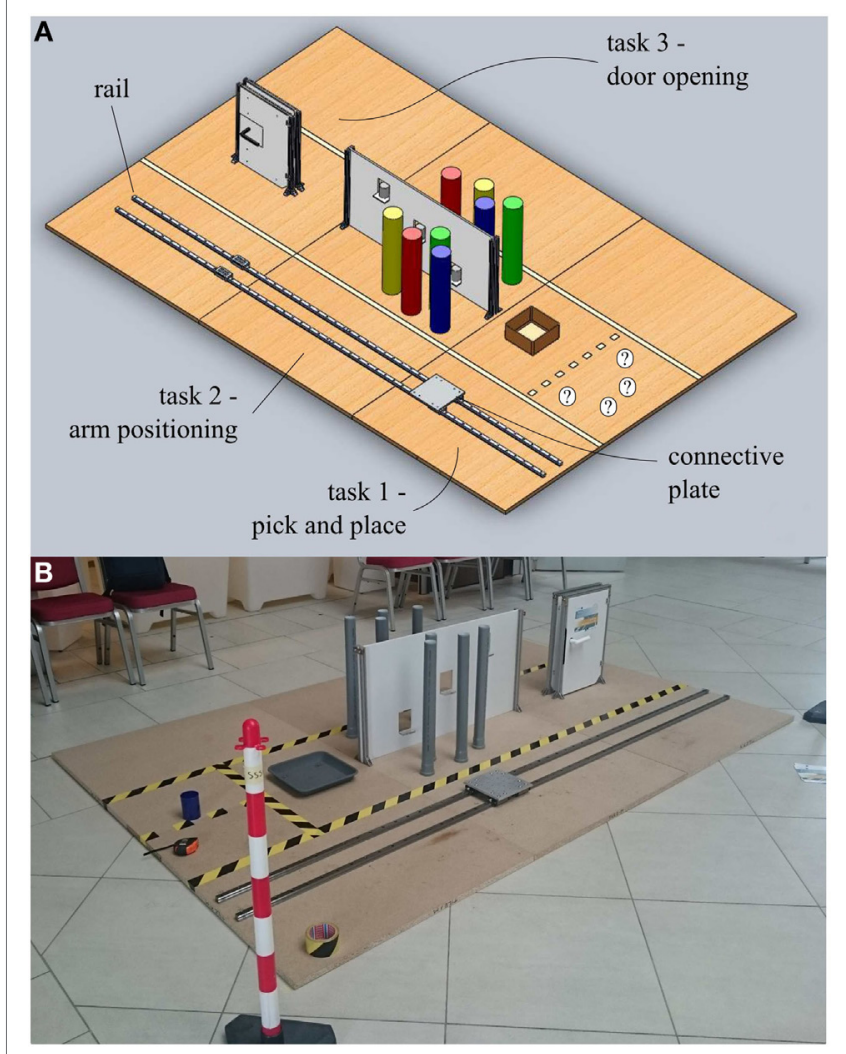

FIGURE 3 | The manipulation scenario was made of three tasks, which can be approached from two sides (with and without a mobile base). Picture (A) shows the CAD model, picture (B) shows the actual implementation.

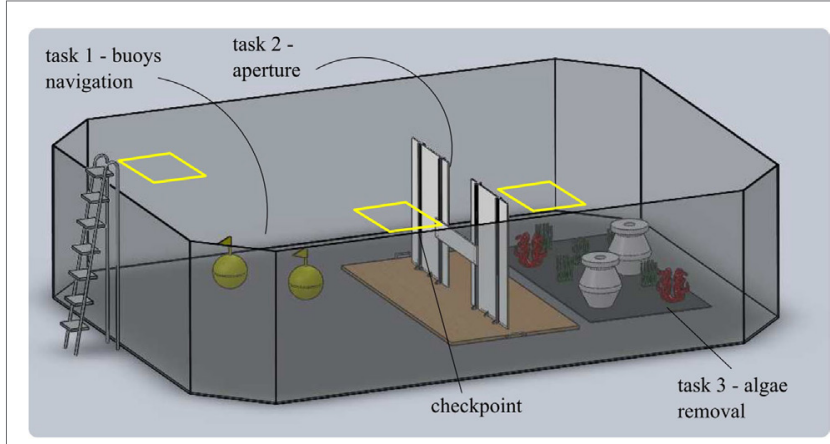

FIGURE 4 | The underwater race was made of three tasks, and checkpoints were positioned at the surface of the water.

in Figure 4. The scoring was defined based on the illustrated tasks, and the challenge development was initiated. The overall design of the fields was revealed to the participants together with challenge rules (Figure 5), so that they were able to replicate the fields for their own convenience and tests: later on, actual commercial materials detailed the design, so that each team was able to actually build a copy of the scenario. 


\section{CHALLENGE DEVELOPMENT, CONTEST RULES, AND SCORING}

The involvement of the teams started with a first phase that was called "expression of interest." During this phase, participants interested in the challenge submitted an intent form that contained the essential information to understand the technical solution proposed and the scenarios which they would like to deal with. This phase was intended to perform a preliminary evaluation on the robots expected during the challenge, and to estimate the number of participants. Once completed, a "pre-selection phase" started, where teams should demonstrate the current development and the envisioned performances of their robots via submitted videos and technical documents. Skills to be shown in the videos were directly related to scenarios and tasks: teams should demonstrate some basic capabilities (forward locomotion and shrinking for locomotion race; picking of objects and arm positioning for manipulation scenario; swimming and shrinking for underwater race) that would allow them to be competitive during the challenge. We judged skills on a do-it base. When a certain skill was not properly shown, we asked for additional videos or explanations, so that further submissions were admitted. It is worth to mention that other challenges (DRC, MBZIRC, UAE Drones for good, etc.) perform evaluation in a one-step fashion, thus merging our expression of interest and pre-selection phases. The additional effort required in splitting the evaluation in two phases is motivated by the novelty of both the scientific field (soft robotics, where technologies are still not well assessed) and the evaluation technique (challenge for soft robots in realistic mock scenarios). For small and new events, such as the RSGC, the expression of interest gives an initial evaluation on the number of participants, acceptance of the challenge, and appropriateness of the tasks. This will mitigate the drawbacks, and allow to perform corrective actions, in case of negative feedback.

Upon evaluation, selected teams were allowed to move to the next phase, which was the "registration phase" where teams actually subscribed for certain scenarios of the challenge. A timetable of the early phases of the challenge development, together with the number of teams is reported in Figure 5, while a diagram of the actual countries which participated in the challenge is presented in Figure 6. It appeared that from the initial number of expression of interests, only one-third of the participants were eventually able to compete in the RSGC (about 20 over 60 ). The expression of interests was almost equally divided among the

\begin{tabular}{|c|c|c|c|c|c|c|c|}
\hline \multicolumn{4}{|c|}{$\underline{2015}$} & \multicolumn{4}{|c|}{$\underline{2016}$} \\
\hline Jun. $30^{\text {th }}$ & Sept. $30^{\text {th }}$ & Oct. $1^{\text {st }}$ & Dec. $31^{\text {th }}$ & Feb. $1^{\text {st }}$ & Mar. $15^{\text {th }}$ & Apr. 29th & Apr. $30^{\text {th }}$ \\
\hline $\begin{array}{l}\text { Rules are } \\
\text { published }\end{array}$ & $\begin{array}{c}\text { Intent form } \\
\text { submission } \\
\text { closes } \\
\uparrow\end{array}$ & $\begin{array}{l}\text { Submission } \\
\text { of the pre- } \\
\text { selection } \\
\text { material } \\
\text { opens }\end{array}$ & $\begin{array}{c}\text { Submission } \\
\text { of pre- } \\
\text { selection } \\
\text { material } \\
\text { closes } \uparrow\end{array}$ & $\begin{array}{c}\text { Admitted } \\
\text { teams } \\
\text { are } \\
\text { natified }\end{array}$ & $\begin{array}{l}\text { Team } \\
\text { registration } \\
\text { closes }\end{array}$ & $\begin{array}{l}\text { RoboSoft } \\
\text { Challenge } \\
\text { Starts } \\
\uparrow\end{array}$ & $\begin{array}{l}\text { RoboSoft } \\
\text { Challenge } \\
\text { Ends }\end{array}$ \\
\hline \multicolumn{2}{|c|}{ Intent } & \multicolumn{2}{|c|}{ Pre-selection } & \multicolumn{2}{|c|}{ Registration } & \multicolumn{2}{|c|}{ Challenge } \\
\hline \multicolumn{4}{|c|}{$\begin{array}{c}\cdot 60 \text { entries received ( } 23 \text { terrestrial race, } \\
21 \text { manipulation, } 16 \text { underwater race) } \\
\text { - } 18 \text { different institutions ( } 8 \mathrm{EU}, 10 \text { extraEU) }\end{array}$} & & & \multicolumn{2}{|c|}{$\begin{array}{l}\text {-19 robots scheduled } \\
\text { (12 terrestrial race, } \\
7 \text { manipulation) }\end{array}$} \\
\hline & & \multicolumn{3}{|c|}{$\begin{array}{l}\text { - } 29 \text { entries received ( } 16 \text { terrestrial race, } \\
11 \text { manipulation, } 2 \text { underwater race) }\end{array}$} & \multicolumn{3}{|c|}{$\begin{array}{l}\cdot 23 \text { entries accepted } \\
\text { (14 terrestrial race, } 9 \text { manipulation) }\end{array}$} \\
\hline
\end{tabular}

FIGURE 5 | The schedule of the challenge organization, with the principal phases and the number of entries each phase highlighted
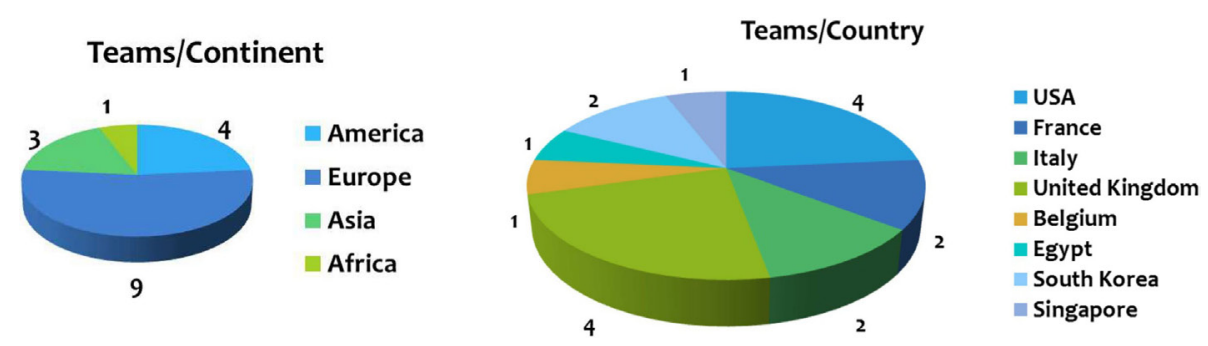

FIGURE 6 | The RSGC raised worldwide interest, with 17 teams participating from 4 different continents which proposed 19 robots. Financial support was provided to selected teams for traveling, accommodation and meals, for a maximum of two members for each selected team. 
three scenarios: 23 entries were for the terrestrial race, 21 for the manipulation, and 16 for the underwater race. Being a novel event targeting a specific subfield of robotics, the initial 60 expression of interests were unexpected (yet welcome): out of these 60 entries, about half only actually submitted the pre-selection material. It is worth to mention that, while the terrestrial and manipulation entries suffered from a moderate reduction, the underwater race dropped from 16 to only 2 entries. Additional six entries were not admitted to the challenge due to the extremely preliminary state of the submitted material, which prevented a proper evaluation: the final pool of 23 participants (submitted for participation into terrestrial race and manipulation) posed already logistic challenges for the competition, which was intended to be performed in 2 days (the first one for testing the robots and the second one for the actual challenge). Four robots retired prior to the beginning of the challenge due to unexpected logistic problems of the teams, so that a final number of 19 robots (submitted from 17 different teams) participated in the challenge: 12 in the terrestrial race and 7 in the manipulation scenario. Although we did not collect data on teams composition, we noticed that teams were mostly made of an experienced researcher (PostDoc) and a few $\mathrm{PhD}$ students. Only one team was from a High School. The dimension of teams varied significantly, ranging from 2 to almost 10 members. Due to a lack of participants, the underwater race was not organized.

The challenge scenarios were implemented via a tile system, i.e., the challenge field was made of several tiles stitched together. As shown in Figures $\mathbf{2}$ and $\mathbf{3}$, each tile represented one task, thus saying that a robot completed a tile is equivalent to say that it completed a task. A tile system has the advantage of providing a flexible implementation, so that the challenge can be extended, reduced, or modified by replacing one tile only. Specific checkpoints were defined inside the scenarios to provide the starting positions for the various trials. A maximum number of three attempts for each task could be performed, after which the task was considered not completed and the robot was moved by the team to the next checkpoint. A fraction of the total points was assigned to the robot in case the task was partially completed. The number of trials required to complete a task also affected the scoring, i.e., the maximum score was earned by completing the task during the first attempt, then the score decreased at each subsequent attempt. Details of the tasks that should be performed, and how the task was considered partially or totally completed, are reported to better understand what the robots were demanded to do.

In the locomotion race, see Figure 2, task 1 was a sand box $\sim 2 \mathrm{~m}$ long and $1 \mathrm{~m}$ wide. It had about $1.5 \mathrm{~cm}$ of sand with fine granularity (i.e., washed and sterilized river sand). From the starting tile to the sand tile, the ground was uneven, thus, a small step of few centimeters was required to enter into and exit from the tile. The task was considered partially solved if the robot reached at least the middle of the tile. The task 2 was a wall tile made of three rigid PVC elements that were moved to reduce the dimension of an aperture. Approximately, the aperture was a square of side $s$, where $s$ was decided by teams prior to the competition. Before starting the terrestrial race, the teams should inform the judges about how challenging the aperture should be for their robot, i.e., to which extent their robot is able to squeeze (or deform), and enter apertures smaller than the nominal robot dimension (with respect to the locomotion direction) $r_{d}$. The more the aperture was reduced, the highest the number of points that was awarded to the robot for correctly negotiating this obstacle. Approximately if $s=r_{d} / 1.1$, tile points were multiplied by 1.1 , if $s=r_{d} / 1.2$ base points were multiplied by 1.2 and so on. This mechanism was crucial, as it highlighted the adaptability of the soft robots, but it could entail gray areas in the regulation. We tried to implement this mechanism in a fair and quantitative manner by resorting to the following rules and considerations. First of all, each team had to declare the locomotion direction of their robots. Judges then measured the frontal projection of the robot with respect to the declared direction. The maximum dimension of the frontal section was then used as $r_{d}$. Robots were evaluated in one of the stances (if robots were able of multi-modal locomotion) used for locomotion, decided by the teams, and robots were allowed to change stance to enter the aperture. Judges supervised and approved the participants evaluation to ensure a fair comparison among different robots and, in case of discrepancies among participants and judges' evaluation, the latter one was used for the calculation of the points (it is worth to mention that only for one robot judges evaluation was conflictual with participants' ones). The task 3 was a stair tile where a two-step stair (step height about $5 \mathrm{~cm}$ ), should be negotiate by moving upward and downward. This is a classic test for robot locomotion, which was considered partially solved if the robot was at least able to climb the stair, and was considered completely solved if the robot was also able to climb down reaching back the ground. Finally, in task 4 , we presented an unstable environment made of rubber tubes held in place by magnets. Rubber tubes represented the collapsible elements of a congested environment. Robots had to navigate through the tubes without dislodging them. As in the wall tile, also in this case the tubes were moved according to the nominal dimension of the robot, $r_{d}$, so that the distance among the tubes was approximately of the same size of the robot. The robot could touch the tubes, but should not push them away from their original location. If up to two tubes were dislodged, the task was considered partially achieved. If more than two tubes were dislodged, the task was considered not achieved. If no tubes were moved, the task was fully solved. It is worth to mention that external cables (e.g., for power supply, pneumatic actuation) were considered part of the robots, which implicitly penalized tethered robots (more prone to dislodging the unstable elements by trapping them with their cables) over untethered ones.

In the manipulation scenario, see Figure 3, the pick and place task (task 1) was made of two subspaces, one (a) where some objects (four different ones) were placed and the other (b) where a collecting basket was fixed. Ten objects were revealed during the testing day, and only four objects were used the day of the challenge. The four objects were: an empty ice cream cone, a wrench, a squared box, and a bottle full of water. The maximum dimension of the objects did not exceed a cubic bounding box of $1000 \mathrm{~cm}^{3}$ in volume and a weight of $0.5 \mathrm{~kg}$. The objects had to be collected inside the basket. Moving an object from side (a) 
to side (b) without succeeding in placing it into the basket was worth a fraction of the points that were earned with a correct placement inside the basket. If an object was damaged during the pick and place operation, the robot did not earn points, no matter if it managed to move the object to the side (b) or even placing the object into the basket. The task 2 was the positioning task (reaching), where robots had to demonstrate their dexterity by touching three cans placed inside small apertures opened in a wall. The tile featured three different lanes from (i) to (iii). Robots should reach the cans by starting from a frontal position with respect to the can they had to touch. This was to prevent robots from starting from lane (i) and touch the can of line (ii), avoiding the corresponding rubber tube (thus, without really negotiating it). The first lane (i) was the simplest one, as the robot can freely elongate to touch the can without the need to negotiate any obstacle. In the second one (ii), a fixed rubber tube was placed between the can and the robot, so that a low degree of dexterity was required to the manipulator. Finally, in lane (iii), there were three rubber tubes between the can and the robot, which had to be negotiated by the manipulator. The aim was to increase the difficulty of the task depending on the lane that was faced. If a manipulator reached beyond the tubes without hitting the target can, the task was considered partially accomplished. Finally, the task 3 was a tile with a small door with a horizontal handle placed at mid height. To open the door, coordination and strength are required. A stable grasp of the handle should be achieved and maintained during the opening manoeuvre. The task was considered completely solved if the handle was turned and the door opened. Task was considered partially completed if the handle was rotated but door was not opened.

The scoring was assigned by judges, supervising the competition both for correctness and assessment, according to the completion criteria specified for each task. Scoring was weighted with

TABLE 2 | Scoring of the terrestrial race.

\begin{tabular}{lllllll}
\hline & Not & Half & Full & $\mathbf{1}^{\circ}$ & $\mathbf{2}^{\circ}$ & $\mathbf{3}^{\circ}$ \\
\hline Task 1 - Sand & 0 & 0.5 & 1 & 0.25 & 0.125 & 0 \\
Task 2 - Aperture & 0 & 2 & 4 & 1 & 0.5 & 0 \\
Task 3 - Stair & 0 & 1 & 2 & 0.5 & 0.25 & 0 \\
Task 4 - Debris & 0 & 2 & 4 & 1 & 0.5 & 0 \\
\hline
\end{tabular}

The points of task 2 were further multiplied by the shrinking factor. Point were assigned for completing partially (half) or totally (full) a task, and additional points were awarded if achieved in the first or second trial.

TABLE 3 | Scoring of the manipulation.

\begin{tabular}{lllllll}
\hline & Not & Half & Full & $\mathbf{1}^{\circ}$ & $\mathbf{2}^{\circ}$ & $\mathbf{3}^{\circ}$ \\
\hline Task 1 - Object 1 handled & 0 & 0.5 & 1 & 0.25 & 0.125 & 0 \\
Task 1 - Object 2 handled & 0 & 0.5 & 1 & 0.25 & 0.125 & 0 \\
Task 1 - Object 3 handled & 0 & 0.5 & 1 & 0.25 & 0.125 & 0 \\
Task 1 - Object 4 handled & 0 & 0.5 & 1 & 0.25 & 0.125 & 0 \\
Task 2 - Can 1 reached & 0 & 0.5 & 1 & 0.25 & 0.125 & 0 \\
Task 2 - Can 2 reached & 0 & 0.75 & 1 & 0.375 & 0.1875 & 0 \\
Task 2 - Can 3 reached & 0 & 1 & 2 & 0.5 & 0.25 & 0 \\
Task 3 - Door opened & 0 & 1.5 & 3 & 0.75 & 0.375 & 0 \\
\hline
\end{tabular}

Points were assigned for completing partially (half) or totally (full) a task, and additional points were awarded if completing the task in the first or second trial. respect to the task difficulties and to the soft skills demonstrated, so that soft-related tasks earned more points than traditional ones. Scoring tables are reported in Tables 2 and 3: the regulation also promoted the successful completion of the tasks, that is scoring full-task in the third attempt resulted in more points than scoring half-task in the first try. The maximum time allowed to complete the scenarios was $20 \mathrm{~min}$ for the locomotion race and $25 \mathrm{~min}$ for the manipulation. After the clearance of each scenario, teams were allowed to move all the necessary equipment to the next scenario, and to check robot functionalities. Each team was allowed to quickly fix, reprogram, and test the robot before the next scenario. However, major hardware modifications were not allowed (i.e., adding or removing parts/tools). Violating this rule resulted in disqualification, but no improper behaviors were reported.

\section{OVERALL CHALLENGE RESULTS}

The RSGC event started with the test drive, the day before the actual challenge, which was intended to allow the teams to test the actual challenge fields and to have a feedback on the robot performance, but no scoring was recorded. Quick robots were able to perform tests in all the scenarios, less fast ones focused instead on specific parts of the challenge, that they considered particularly challenging. All robots except four (two in manipulation, the others in locomotion) performed the test drive. Two of the robots that did not benefit from the test drive suffered from severe malfunctioning that prevented them from participating to the actual challenge: nevertheless they were allowed to perform demonstrations of specific part of the scenarios; the other two were able to recover from the failure and participate in the challenge. During the day of the challenge, three more robots suffered from unexpected malfunctioning, which resulted in a severe decrease of the performance for two robots (one in manipulation, one in locomotion) and in a small decrease for the third (in locomotion). A fourth one experienced a severe malfunctioning but it was quickly fixed, so that the failure did not influence the performance of the robot. The overall score for the terrestrial and manipulation scenarios are presented in Table 4, while in Tables 5 and $\mathbf{6}$ tasks results are presented. The winning robots are depicted in Figure 7: the Grand Challenge winner, that is the robot that earned the highest number of points participating and scoring in all scenarios, was an origamiwheeled vehicle featuring a flexible arm and pincher (ranking second in the locomotion race, and first in the manipulation scenario); the locomotion race was ruled by a wheeled robot with inflatable air chambers; and the manipulation scenario was won by a pneumatic fiber-reinforced gripper (actually, this robot ranked second after the Grand Challenge winner, but since a robot could not be awarded twice by regulation, the robot shifted to the winning position).

Results for the locomotion race presented significant variability: two robots performed the whole scenario and earned the same amount of points, so that the winner was decided by the completion time, while four robots did not perform even the simplest tile, Table 4. Among the latter, two were severely impaired, so that their bad performance could not be related to specific 
technical solutions implemented; the remaining two based the locomotion on ground friction, so that their performance was significantly affected by the difference between their testing surface and the actual substrate of the challenge. Noticeably, the robot that ranked sixth in the final scoring, Table 4, had a similar friction problem experienced during the day of the trial: that team worked overnight to fix the problem by changing the skin of their robot, and they moved from a null to a reasonably good score. Participating robots performed effectively at least three out the four proposed tasks: sand, aperture, and unstable elements tiles were performed by almost all the robots, Table 5, with a normalized mean score of about 0.5 , which rises to a significant 0.7 if only the robots who actually scored some points are considered (normalization with respect to the maximum score). Although robots seemed able to tackle soft-related tasks easily, mean score in the third task (stairs) dropped to about 0.3 (0.4 if considering

TABLE 4 | Results of the terrestrial race and manipulation scenario.

\begin{tabular}{lccccc}
\hline \multirow{2}{*}{ Position } & \multicolumn{2}{c}{ Terrestrial } & & \multicolumn{2}{c}{ Manipulation } \\
\cline { 2 - 3 } \cline { 5 - 6 } & Score & Time & & Score & Time \\
\hline 1 & 15.4 & $2: 33$ & & 14.3 & $20: 26$ \\
2 & 15.4 & $7: 50$ & & 10.6 & $23: 55$ \\
3 & 13.3 & $16: 10$ & & 9.1 & $24: 49$ \\
4 & 8.8 & $19: 46$ & & 6.9 & $24: 53$ \\
5 & 8.6 & $7: 57$ & & 6.4 & $25: 00$ \\
6 & 8.6 & $15: 30$ & & 5.2 & $24: 51$ \\
7 & 8.4 & $18: 46$ & & 0.8 & $25: 00$ \\
8 & 7.2 & $20: 00$ & & \\
9 & 0 & - & & \\
10 & 0 & - & & \\
11 & 0 & - & & \\
12 & 0 & - & & \\
Mean & 7.1 & & 7.6 & \\
\hline
\end{tabular}

Time is reported in the format mm:ss. the subset of scoring robots), and was performed by three robots only, Table 5.

Results for the manipulation scenario present a lower variability than locomotion ones (see SD bars in Figure 8), especially considering that no robot scored 0 , and that the last robot in the ranking, Table 4, stopped working right after the beginning of the trial. Operational time is much higher than for locomotion, and some robot did not finish the track due to a lack of time rather than a lack of capabilities. However, the intra-task variability was much higher, Table 6: the pick and place task was easily performed for the fragile object (ice cream cone, task 1.1), while the other objects were slightly more difficult. However, most of the robots started from the door and the positioning tiles, and gave up due to the lack of time for the pick and place task. With respect to the positioning tile, it appeared that the most challenging part was the third line (Figure 3), where three vertical tubes stood in between the robot and the target: the mean score drops dramatically from the 0.91 and 0.8 of the first two lines to 0.37 of the third line.

Finally, we compared our results with the data available for other task-related challenges, specifically with the Darpa Robotic Challeng (DRC) and the euRathlon (data are available from corresponding websites). DRC is a task-oriented challenge that features legged vehicles which should perform complex tasks in a congested environment; while the euRathlon challenges underwater, ground and air vehicles in exploration and interaction tasks: we consider such challenge of comparable difficulties in the respective, different fields. We reported both trials and final score of the DRC, while for the euRathlon were selected scenarios where at least five teams participated; thus, the Land-Air-Sea scenario, the Land 1 and Land 2 scenarios, the Sea 1 and Sea 2 scenarios, and the Air 1 . The data were normalized with respect to the maximum score, and then they were reported in Figure 8: results are characterized by mean values included between 0.3

TABLE 5 | Results of the terrestrial race, expanded by tasks.

\begin{tabular}{|c|c|c|c|c|c|c|c|c|c|c|c|c|c|c|}
\hline & \multicolumn{12}{|c|}{ Score } & \multirow{2}{*}{$\begin{array}{c}\text { Mean } \\
0.58\end{array}$} & \multirow{2}{*}{$\begin{array}{c}\text { nMean } \\
0.47(0.7)\end{array}$} \\
\hline Task 1 & 1.25 & 1.25 & 0.75 & 0.75 & 1.13 & 1.13 & 0 & 0.75 & 0 & 0 & 0 & 0 & & \\
\hline Task 2 & 6.67 & 6.67 & 7.5 & 5 & 0 & 4.5 & 5.36 & 6.41 & 0 & 0 & 0 & 0 & 3.51 & $0.47(0.7)$ \\
\hline Task 3 & 2.5 & 2.5 & 0 & 0 & 2.5 & 0 & 0 & 0 & 0 & 0 & 0 & 0 & 0.63 & $0.25(0.38)$ \\
\hline Task 4 & 5 & 5 & 5 & 3 & 5 & 3 & 3 & $0^{a}$ & $0^{\mathrm{b}}$ & $0^{a}$ & 0 & 0 & 2.42 & $0.48(0.73)$ \\
\hline
\end{tabular}

Scores identified by arepresent robots experiencing severe malfunctioning during the race, while before the contest. Mean and normalized-mean values are reported, while in brackets the normalized-mean is considered only for the robot who actually earned some points.

TABLE 6 | Results of the Manipulation scenario, expanded by tasks.

\begin{tabular}{|c|c|c|c|c|c|c|c|c|c|}
\hline & & & & Score & & & & Mean & nMean \\
\hline Task 1.1 & 1.25 & 1.25 & 0 & 0 & 1.13 & 0 & 0 & 0.52 & 0.41 \\
\hline Task 1.2 & 1.25 & 0 & 0 & 1.13 & 0 & 0 & 0 & 0.34 & 0.27 \\
\hline Task 1.3 & 1.25 & 1.25 & 0 & 0 & 0 & 0 & 0 & 0.36 & 0.29 \\
\hline Task 1.4 & 1.13 & 1.25 & 0 & 0 & 0 & 0 & 0 & 0.34 & 0.27 \\
\hline Task 2.1 & 1.25 & 1.25 & 1 & 1.25 & 1.13 & 1.25 & 0.75 & 1.13 & 0.9 \\
\hline Task 2.2 & 1.88 & 1.88 & 1.88 & 1.5 & 1.88 & 1.69 & 0 & 1.53 & 0.81 \\
\hline Task 2.3 & 2.5 & 0 & 2.5 & 1.5 & 0 & 0 & 0 & 0.93 & 0.37 \\
\hline Task 3 & 3.75 & 3.75 & 3.75 & 1.5 & 2.25 & $2.25^{b}$ & $0^{\mathrm{a}}$ & 2.46 & 0.66 \\
\hline
\end{tabular}

Scores identified by arepresent robots experiencing malfunctioning during the race, while bdenotes malfunction before the contest. 

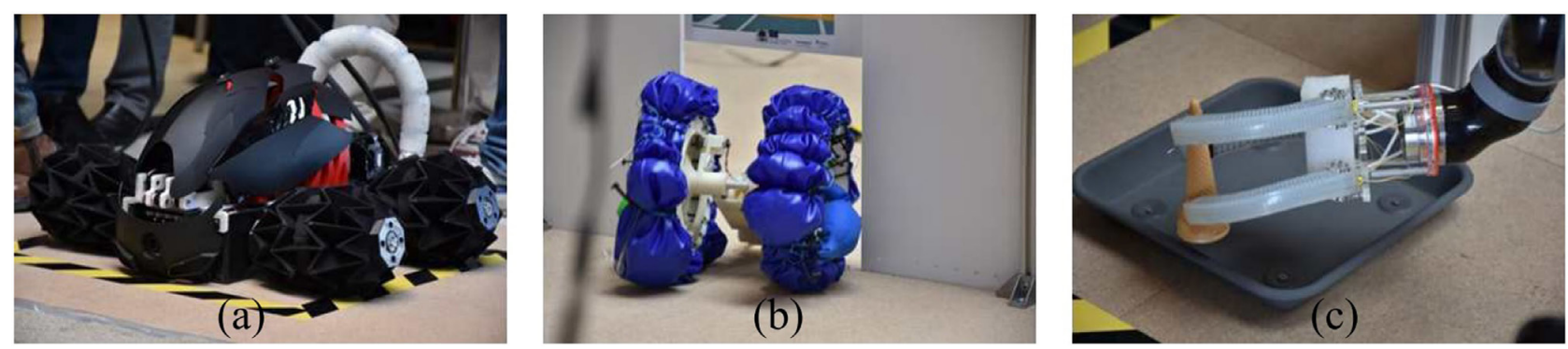

FIGURE 7 | The Grand Challenge winner (A) had origami wheels and a flexible manipulating arm (white, in the back). The locomotion race winner (B) had inflatable air-chambers attached to a wheeled device, while the manipulation winner (C) featured a pneumatic three-finger gripper.

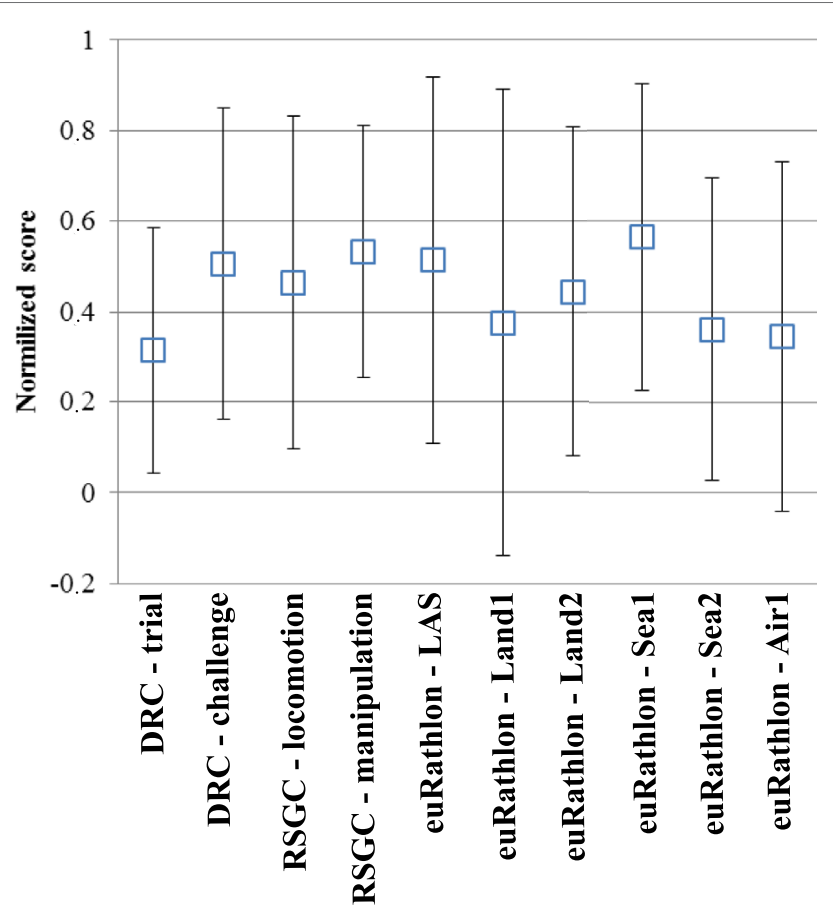

FIGURE 8 | Comparison among task-oriented challenge results (normalized with respect to the maximum score being achieved). All results are included between 0.3 and 0.6 , with the majority of them between 0.4 and 0.6 .

and 0.6, with most of the data included between 0.4 and 0.6. DRC challenge results greatly improved with respect to the trial ones, passing from the $0.3-0.4$ interval to the $0.4-0.6$. SD of the results, illustrated as the error bars in the picture, is also similar for all the challenges: the smallest one was obtained for the DRC trial, while the highest was derived from the Land 1 data of the euRathlon competition.

\section{DISCUSSION AND LESSONS LEARNT}

By analyzing the amount of submitted entries (intent forms), we considered that the RSGC was timely organized, and it hit a fertile discipline of robotics, which still requires standardization and benchmarking. The initial distribution of entries among the three proposed scenarios confirmed also that the application fields were correctly envisaged by the organizers and in literature (Kim et al., 2013; Rus and Tolley, 2015); however, the abrupt drop in the underwater submissions (pre-selection material) outnumbered the expected physiological decrease experienced by terrestrial and manipulation entries (Figure 5). Although we have no feedback to understand this reduction, we hypothesize two reasons for that: the first one is that state of the art underwater soft robots are still very far from realworld application standards. This could be related to the main motivation behind the development of underwater soft robots, which is most of the time the analysis of biological models to gather fundamental insights about behaviors or locomotion. An example is the octopus-inspired arm developed in the framework of the OCTOPUS project (Laschi et al., 2012), which included biological insights about the muscular structure of the animal, and that was further developed in an application-driven prototype, by abstracting the anatomy of the animal but obtaining a more reliable device (Ranzani et al., 2016). The second reason is that underwater robotics poses additional constrains on communication, control, and design: wireless communication via traditional means (e.g., radio frequency or wi-fi) is not possible underwater, thus, it requires acoustic modems or tethered systems; state of soft robots control is still not adequate to properly operate underwater robots (which requires to take into account effects arising from drag, buoyancy, added masses, etc.); finally, all devices should be carefully insulated in order to work underwater and avoid rapid degradation of the prototypes or the catastrophic failure of the robot. It is possible that some of the teams that expressed interest for the underwater race did underestimate the complexity associated with designing and controlling a soft robots for aquatic operation, which requires a number of additional technical skills and facilities.

Although quantitative data are not available, it is also possible that this reduction was associated to the complexity of the underwater tasks that the robots were demanded to solve during the challenge. The underwater competition scenario was indeed slightly more challenging than the others. Task 1 required $3 \mathrm{D}$ goal directed locomotion (hitting a number of buoys placed at different depths). Task 2 required the robot 
to squeeze into an aperture, with the additional dare of doing so underwater (where robot control and/or teleoperation is more challenging). Task 3 required to navigate an unstable environment, with the added challenge of performing some kind of manipulation in order to selectively eradicate a number of objects (mimicking algae). In future editions, this scenario could be very easily simplified in order to increase participation, e.g., by proposing planar locomotion and by reducing manipulation efforts.

These argumentations should not suggest that, on the contrary, developing a reliable terrestrial soft robot is a far easier task with state of the art technology. Especially for locomotion, we noticed a great variety of different solutions, comprising wheeled systems, legged robots (from four to six legs), rolling ones, robots based on vibration, origami robots, snake-like ones, reconfigurable robots, worm-inspired robots, and even ameba-inspired ones. All this variety was exposed to a even more vast number of different failures, comprising (but not limited to) leakage of air in pneumatic systems, explosion of air chambers, detachment of tendons, mechanical failure of the supporting structures, and detachment of electric cables from soft bodies. The deployment and the repeated, reliable use of soft robots appears to be still a significant challenge, coherently with the relatively young history of this field and the small number of real-world devices currently in use. With respect to manipulation, we noticed two main approaches to the challenge: one where the robot had a clear distinction between a rigid arm and a soft gripper; the second where the whole arm is flexible and soft. The latter approach had the potential to solve the whole tasks of the scenario, as the Grand Challenge winner did: conversely the manipulation winner completely failed positioning task 2.1 (Table 6) but performed very well in all the other tasks. In general, it appeared that the soft gripper technologies are already adaptable, effective solutions which can be employed jointly with traditional components. As a critic recap of the state of the art of soft robotics we can say that, in locomotion, soft robots demonstrated augmented, focused capabilities with respect to the soft-related tasks, such as delicate contact and adaptability of the body: on the other hand, they still have to demonstrate their effectiveness on traditional complex tasks. It is worth to mention that the stair tile (task 3 ) was extremely challenging for the robots (Table 5). This could be related to the fact that gaits traditionally used in soft locomotion (e.g., crawling, inching, rolling, etc...) inherently suffer from high obstacles. One solution could be to perform multi-modal locomotion, changing from a crawling to a climbing gait when required. However, this aspect remains unchallenged by the vast majority of soft robots in the state of the art. In manipulation, flexible arms and deformable grippers demonstrated exciting progress both on soft-related tasks, such as delicate manipulation and adaptive arm positioning, and in traditional tasks, such as pick and place and door opening.

By analyzing the robots participating in the challenge, sometimes we noticed what we called a "reductionist design." With this term, we mean the design process that develops a simplified yet effective robot purposely suited for the event and the scoring system: while this is perfectly correct and sometimes even smarter than producing more complex designs, it renounces to one of the fundamental goals of robotic challenges, which is to push forward the state of the art. This is a fault of task-oriented challenges, which allow turnarounds with out-of-the-box solutions: as in the DRC not walking was a big advantage (Guizzo and Ackerman, 2015), similarly hybrid soft-rigid solutions performed amazingly in the overall RSGC. With this respect, we consider the first objective of the challenge only partially achieved. As a matter of fact, we notice small progresses beyond the state of the art. Presented solutions were mostly optimizations or refinements of current soft technologies. However, this result was in line with the scale (related to prizes, size of community, and novelty of the field) of the proposed challenge, so that disruptive innovations were really unlikely to be developed. Greater monetary prizes and focused objectives could further progress the technologies of the field, while the RSGC proved that a lively community of soft roboticists is ready to compete at the edge of the state of the art.

It is worth to mention, however, that the overall performances of the robots were in line with other task oriented challenges, and difficulty seemed appropriate to include enough technical improvements without proposing unattainable tasks. Soft robotics seems mature and flexible enough to answer to specific robotic duties in realistic mock scenarios. By the effective completion of most of the proposed tasks, RSGC proved that soft solutions (from design to fabrication) are ready to be part of the innovation chain, and that they can be tailored to tackle specific niches. From the results of the competition, it appears that all teams were able to steer the development of their soft robots toward the accomplishment of traditional and innovative robotic tasks.

The RSGC provided a useful benchmarking tool, which allowed to highlight the performance and limitation of current soft robots. Some technical solutions clearly emerged as predominant for certain tasks. For example, even if soft locomotion features extremely different gaits (legged, crawling, inching, serpentine, rolling, etc.) deformable wheels greatly outperformed other solutions with respect to the speed of locomotion. Moreover, we noticed that sophisticated gaits and actuation systems were usually not underpinned by refined control and sensing systems. Mostly, they relied on open-loop controls or on operators, which however proved not to be enough effective on the presented scenarios. From the first edition of the RSGC, it is not possible to properly rate soft technologies, and it is also beyond the scope of this paper. The number of participant robots and the trials are still low to produce a meaningful statistic on what technology is best suited for a certain task. However, from RSGC clearly emerged that is possible to quantitatively compare performance of soft robots and, to date, RSGC is the only challenge that assess performance of soft robots on realistic scenarios. While single components (actuators, algorithms, sensors, etc.) are already evaluated by means of quantitative criteria, RSGC was the first attempt to benchmark whole soft robotics systems. Further editions or similar contests could enlarge the sample and highlight predominant solutions, so that more insights could be provided to soft robotics researchers. 
In conclusion, contests, such as the RSGC, proved to be effective in benchmarking and steering soft technologies, while they appear to have limited impact on pushing forward the state of the art. We envisage that soft robotics contests, with tasks tailored on specific societal and industrial needs, and with greater monetary prizes than the one proposed in the RSGC, will be proficient methodologies to boost the novel field of soft robotics.

\section{AUTHOR CONTRIBUTIONS}

All authors conceived the first version of the rules, defined the scenarios and tasks at conceptual level. MCal refined the rules, designed and assembled the competition fields, and coordinated the logistic of the challenge, including the collection and elaboration of the data. FC managed the proposal submission, MM and MCian helped with logistic. MCal and CL analyzed the results

\section{REFERENCES}

Arienti, A., Calisti, M., Serchi, F. G., and Laschi, C. (2013). "Poseidrone: design of a soft-bodied ROV with crawling, swimming and manipulation ability," in Proceedings of the MTS/IEEE OCEANS (San Diego: MTS/IEEE), 1-7.

Belanger, J. H., Bender, K. J., and Trimmer, B. A. (2000). Context dependency of a limb withdrawal reflex in the caterpillar Manduca sexta. J. Comp. Physiol. A 186, 1041-1048. doi:10.1007/s003590000161

Calisti, M., Falotico, E., and Laschi, C. (2016). Hopping on uneven terrains with an underwater one-legged robot. IEEE Robot. Autom. Lett. 1, 461-468. doi:10.1109/LRA.2016.2521928

Chrispell, J., Fauci, L., and Shelley, M. (2013). An actuated elastic sheet interacting with passive and active structures in a viscoelastic fluid. Phys. Fluids 25, 013103. doi:10.1063/1.4789410

Cianchetti, M., Calisti, M., Margheri, L., Kuba, M., and Laschi, C. (2015). Bioinspired locomotion and grasping in water: the soft eight-arm octopus robot. Bioinspir. Biomim. 10, 035003. doi:10.1088/1748-3190/10/3/035003

Cianchetti, M., Ranzani, T., Gerboni, G., Nanayakkara, T., Althoefer, K., Dasgupta, P., et al. (2014). Soft robotics technologies to address shortcomings in today's minimally invasive surgery: the stiff-flop approach. Soft Robot. 1, 122-131. doi:10.1089/soro.2014.0001

Cicconofri, G., and DeSimone, A. (2015). A study of snake-like locomotion through the analysis of a flexible robot model. Proc. Math. Phys. Eng. Sci. 471, 20150054. doi:10.1098/rspa.2015.0054

Clark, A. J., Tan, X., and McKinley, P. K. (2015). Evolutionary multiobjective design of a flexible caudal fin for robotic fish. Bioinspir. Biomim. 10, 065006. doi:10.1088/1748-3190/10/6/065006

Deashapriya, K., Sampath, P., Wijekoon, W., Jayaweera, N., and Kulasekera, A. (2016). "Biomimetic flexible robot arm design and kinematic analysis of a novel flexible robot arm," in Moratuwa Engineering Research Conference (MERCon) (Moratuwa: IEEE), 385-390.

Deng, Z., Stommel, M., and Xu, W. (2016). A novel soft machine table for manipulation of delicate objects inspired by caterpillar locomotion. IEEE/ASME Trans. Mechatron. 21, 1702-1710. doi:10.1109/TMECH.2016.2519333

Dias, J., Althoefer, K., and Lima, P. U. (2016). Robot competitions: What did we learn? [competitions]. IEEE Robot. Autom. Mag 23, 16-18. doi:10.1109/ MRA.2015.2511678

Elango, N., and Faudzi, A. A. M. (2015). A review article: investigations on soft materials for soft robot manipulations. Int. J. Adv. Manuf. Technol. 80, 1027-1037. doi:10.1007/s00170-015-7085-3

Fakhari, A., Keshmiri, M., Kao, I., and Jazi, S. H. (2016). Slippage control in soft finger grasping and manipulation. Adv. Robot. 30, 97-108. doi:10.1080/01691 864.2015.1105149

Ferri, G., Ferreira, F., and Djapic, V. (2015). "Boosting the talent of new generations of marine engineers through robotics competitions in realistic environments: the sauc-e and eurathlon experience," in OCEANS 2015 (Genova: IEEE), 1-6. and discussed further developments. All authors contributed to the writing of the paper.

\section{ACKNOWLEDGMENTS}

Authors would like to thanks Ms. Irene Mannari for the logistic and design support, Mr. Izad Tamadon for multi-media contents, and Dr. Laura Margheri for the financial management. The RoboSoft Grand Challenge initiative was firstly suggested by Prof. Adriana Tapus.

\section{FUNDING}

This work was funded by the European Commission within the framework of the RoboSoft Coordination Action, under the contract FP7-ICT-2013-C project \# 619319.

Fiazza, C., Salumäe, T., Listak, M., Kulikovskis, G., Templeton, R., Akanyeti, O., et al. (2010). "Biomimetic mechanical design for soft-bodied underwater vehicles," in OCEANS 2010 IEEE (Sydney: IEEE), 1-7.

Guizzo, E., and Ackerman, E. (2015). The hard lessons of Darpa's robotics challenge [news]. IEEE Spectr 52, 11-13. doi:10.1109/MSPEC.2015.7164385

Jayaram, K., and Full, R. J. (2016). Cockroaches traverse crevices, crawl rapidly in confined spaces, and inspire a soft, legged robot. Proc. Natl. Acad. Sci. U.S.A 113, E950-E957. doi:10.1073/pnas.1514591113

Katzschmann, R. K., Marchese, A. D., and Daniela, R. (2015). Autonomous object manipulation using a soft planar grasping manipulator. Soft Robot. 2, 155-164. doi:10.1089/soro.2015.0013

Kim, K., Agogino, A. K., Moon, D., Taneja, L., Toghyan, A., Dehghani, B., et al. (2014). "Rapid prototyping design and control of tensegrity soft robot for locomotion," in IEEE International Conference on Robotics and Biomimetics (ROBIO) (Hanoi: IEEE), 7-14.

Kim, S., Laschi, C., and Trimmer, B. (2013). Soft robotics: a bioinspired evolution in robotics. Trends Biotechnol. 31, 287-294. doi:10.1016/j.tibtech.2013.03.002

Kitano, H., Asada, M., Noda, I., and Matsubara, H. (1998). Robocup: robot world cup. IEEE Robot. Autom. Mag. 5, 30-36. doi:10.1109/100.728221

Laschi, C., and Cianchetti, M. (2014). Soft robotics: new perspectives for robot bodyware and control. Front. Bioeng. Biotechnol. 2:3. doi:10.3389/fbioe.2014.00003

Laschi, C., Mazzolai, B., Cianchetti, M., Margheri, L., Follador, M., and Dario, P. (2012). Soft robot arm inspired by the octopus. Advanced Robotics (Special Issue on Soft Robotics) 26, 709-727. doi:10.1163/156855312X626343

Li, H., Go, G., Ko, S. Y., Park, J.-O., and Park, S. (2016). Magnetic actuated ph-responsive hydrogel-based soft micro-robot for targeted drug delivery. Smart Mater. Struct. 25, 027001. doi:10.1088/0964-1726/25/2/027001

Lima, P. U., Nardi, D., Kraetzschmar, G., Berghofer, J., Matteucci, M., and Buchanan, G. (2014). Rockin innovation through robot competitions [competitions]. IEEE Robot. Autom. Mag. 21, 8-12. doi:10.1109/MRA.2014.2314015

Lin, H., Leisk, G., and Trimmer, B. (2013). Soft robots in space: a perspective for soft robotics. Acta Futura 6, 69-79. doi:10.2420/ACT-BOK-AF

Manti, M., Hassan, T., Passetti, G., D’Elia, N., Laschi, C., and Cianchetti, M. (2015). A bioinspired soft robotic gripper for adaptable and effective grasping. Soft Robot. 2, 107-116. doi:10.1089/soro.2015.0009

Masters, W. A., and Delbecq, B. (2008). Accelerating Innovation with Prize Rewards: History and Typology of Technology Prizes and a New Contest Design for Innovation in African Agriculture. International Food Policy Research Institute (IFPRI). IFPRI discussion papers 835.

Mezoff, S., Papastathis, N., Takesian, A., and Trimmer, B. A. (2004). The biomechanical and neural control of hydrostatic limb movements in manduca sexta. J. Exp. Biol. 207, 3043-3053. doi:10.1242/jeb.01136

Murphy, R. R. (2000). Using robot competitions to promote intellectual development. AI Magazine 21. doi:10.1609/aimag.v21i1.1497

Ranzani, T., Cianchetti, M., Gerboni, G., Falco, I. D., and Menciassi, A. (2016). A soft modular manipulator for minimally invasive surgery: design and 
characterization of a single module. IEEE Trans. Robot. 32, 187-200. doi:10.1109/TRO.2015.2507160

Riener, R., and Seward, L. J. (2014). "Cybathlon 2016," in IEEE International Conference on Systems, Man, and Cybernetics (SMC) (Budapest: IEEE), 2792-2794.

Rus, D., and Tolley, M. T. (2015). Design, fabrication and control of soft robots. Nature 521, 467-475. doi:10.1038/nature14543

Schneider, F. E., Wildermuth, D., and Wolf, H. L. (2015). "Elrob and eurathlon: improving search amp; rescue robotics through real-world robot competitions," in 10th International Workshop on Robot Motion and Control (RoMoCo) (Poznan, Poland), 118-123.

Seok, S., Onal, C., Kyu-Jin, C., Wood, R., Rus, D., and Sangbae, K. (2013). Meshworm: a peristaltic soft robot with antagonistic nickel titanium coil actuators. IEEE/ASME Trans. Mechatron. 18, 1485-1497. doi:10.1109/TMECH.2012. 2204070

Shepherd, R., Ilievski, F., Choi, W., Morin, S., Stokes, A., Mazzeo, A., et al. (2011). Multigait soft robot. Proc. Natl. Acad. Sci. U.S.A 108, 20400-20403. doi:10.1073/ pnas. 1116564108

Shintake, J., Rosset, S., Schubert, B., Floreano, D., and Shea, H. (2016). Versatile soft grippers with intrinsic electroadhesion based on multifunctional polymer actuators. Adv. Mater. Weinheim 28, 231-238. doi:10.1002/adma. 201504264

SoftRoboticsToolkit. (2016). Available at: http://softroboticstoolkit.com/

Song, S.-H., Kim, M.-S., Rodrigue, H., Lee, J.-Y., Shim, J.-E., Kim, M.-C., et al. (2016). Turtle mimetic soft robot with two swimming gaits. Bioinspir. Biomim. 11, 036010. doi:10.1088/1748-3190/11/3/036010

Steltz, E., Mozeika, A., Rodenberg, N., Brown, E., and Jaeger, H. (2009). “Jsel: jamming skin enabled locomotion," in IEEE/RSJ Int. Conf. on Intelligent Robots and Systems (IEEE/RSJ), 5672-5677.

Sun, Y., Song, S., Liang, X., and Ren, H. (2016). A miniature soft robotic manipulator based on novel fabrication methods. IEEE Robot. Autom. Lett. 1, 617-623. doi:10.1109/LRA.2016.2521889
Trivedi, D., Rahn, C. D., Kier, M., and WalkerD, I. D. (2008). Soft robotics: biological inspiration, state of the art, and future research. Appl. Bionics Biomech. 5, 99-117. doi:10.1155/2008/520417

Umedachi, T., Vikas, V., and Trimmer, B. A. (2016). Softworms: the design and control of non-pneumatic, 3d-printed, deformable robots. Bioinspir. Biomim. 11, 025001. doi:10.1088/1748-3190/11/2/025001

Urai, K., Sawada, R., Hiasa, N., Yokota, M., and DallaLibera, F. (2015). Design and control of a ray-mimicking soft robot based on morphological features for adaptive deformation. Artif. Life Robot. 20, 237-243. doi:10.1007/ s10015-015-0216-y

Wyffels, F., Bruneel, K., Kindermans, P.-J., D’Haene, M., Woestyn, P., Bertels, P., et al. (2011). "Robot competitions trick students into learning," in Proceedings of the 2nd International Conference on Robotics in Education, RiE (Singapore), 47-52.

Yu, X., and Iida, F. (2014). Minimalistic models of an energy-efficient vertical-hopping robot. IEEE Trans. Ind. Electron. 61, 1053-1062. doi:10.1109/ TIE.2013.2266080

Conflict of Interest Statement: The authors declare that the research was conducted in the absence of any commercial or financial relationships that could be construed as a potential conflict of interest.

The reviewer LN and handling editor declared their shared affiliation, and the handling editor states that the process nevertheless met the standards of a fair and objective review.

Copyright (C) 2016 Calisti, Cianchetti, Manti, Corucci and Laschi. This is an open-access article distributed under the terms of the Creative Commons Attribution License (CC BY). The use, distribution or reproduction in other forums is permitted, provided the original author(s) or licensor are credited and that the original publication in this journal is cited, in accordance with accepted academic practice. No use, distribution or reproduction is permitted which does not comply with these terms. 